\title{
Process variation in Selective Laser Melting of Ti-6Al-4V alloy
}

\author{
Zhuoer Chen ${ }^{1,2}$, Caroline Lee ${ }^{2}$, Sheng $\mathrm{Cao}^{1,3}$, Xuerui $\mathrm{Lyu}^{3}$, Xinhua $\mathrm{Wu}^{1,3}$, Chris Davies ${ }^{1,2}$ \\ 1. Monash Centre for Additive Manufacturing, 15-17 Normanby Rd, Notting Hill, VIC 3168, Australia. \\ 2. Department of Mechanical and Aerospace Engineering, Monash University, Clayton, VIC 3168, Australia. \\ 3. Department of Materials Science and Engineering, Monash University, Clayton, VIC 3168, Australia.
}

\begin{abstract}
The present work explores the variation in Ti-6Al-4V part quality introduced by the key process operations of Selective Laser Melting (SLM) process, the recoating, the gas flow, and the laser beam irradiation. Novel specimens and experiments were designed to characterize the differences in surface quality and thermal history as a function of part geometry and location on the build platform. The variation in the roughness of inclined surfaces shows a clear dependency on the laser incidence angle and the influence of gas flow on process by-products. The direction in which the laser beam traverse across the build area with respect to the gas flow direction also affects the surface quality. Thermal profiles were recorded by attaching thermocouples to the surface of the built part with various geometries. The measured temperature profiles show intense local fluctuations due to the rapid movement of the laser beam. The parts also experience a continuous heat treatment throughout the SLM process due to the low effective conductivity of the powder bed and continuous heat input by the laser.
\end{abstract}

\section{Introduction}

Selective Laser Melting (SLM) is an additive manufacturing process that employs a focused laser beam to fuse consecutive layers of powder particles to form three-dimensional parts. The SLM technology is one of the most prominent metal additive manufacturing (AM) methods, and is advantageous in making small batches of complex shaped components with high resolution and relatively lower surface roughness as compared to other metal AM variants such as Directed Light Fabrication (DLF) and Electron Beam Melting (EBM) technique.

The high variability in part quality defined by dimensional accuracy, mechanical and physical properties still remains a hurdle for qualification of metal AM parts and processes [1]. Expensive trial-and-error experiments are usually necessary to generate statistically reliable database for replicating high-performance components, while efforts can be made by numerical modelling and on-line monitoring of the process.

Various numerical simulation methods has been employed to correlate the melt pool geometry, size and dynamics with AM processing parameters to better fundamental understanding of the process and to reduce the trial-and-error experimental cost. Lattice Boltzmann method [2], Volume of Fluid (VOF) method [3][4], Computational Fluid Dynamic method [5] and hybrid method [6] take into account some or all of the effects of surface tension, evaporation, Marangoni convection and stochastic powder to better understand the formation mechanisms of SLM-specific defects such as spatter and keyholes. The estimations of melt pool temperature distribution and dimensions with improved accuracy as outcomes of the aforementioned models of these models can also be used as inputs for Finite Element models which are computationally more efficient for scaling up in order to predict thermal stress from temperature field simulation.

On-line monitoring devices that collect the information of the build during the SLM process were developed for measurement and observation of melt pool size [7], powder bed re-coating error [8], thermal distortion [9], damage to re-coating blades [8] and melt pool dynamics [10][11]. The information acquired through on-line monitoring can then be used as a feedback in a closed-loop control to improve the part quality.

The current work addresses the variability in part quality though experimental investigation with novel design of experiments. The variation in surface roughness across the build plate of a commercial SLM machine Concept Laser X-line 1000R is analysed from the perspective of machine related process inconsistency. Furthermore, the temperature profiles were recorded in real time during SLM process from designed locations in the specimens of varied geometry. The variance in thermal history across a large complex component adds another dimension to the process variation.

\section{Materials and Experiments}

\section{Powder material}

The feedstock powder used was gas atomised Ti-6Al-4V powder with D10, D50 and D90 of $16 \mu \mathrm{m}, 30 \mu \mathrm{m}$ and $50 \mu \mathrm{m}$.

\section{The SLM process}

The SLM experiments were carried out using an EOS M280 machine and a Concept Laser X-line 1000R machine. Before the SLM process start, the substrate was heated up to $100^{\circ} \mathrm{C}$ and the build chamber was pre-flooded with Argon gas until the oxygen content reached below 1000 ppm.

(C) The Authors, published by EDP Sciences. This is an open access article distributed under the terms of the Creative Commons Attribution License 4.0 (http://creativecommons.org/licenses/by/4.0/). 
Sample placement on build platform

15 cubic samples with a dimension of $10 \mathrm{~mm} \times 10 \mathrm{~mm} \times 15 \mathrm{~mm}$ were placed on a build platform of Concept Laser X-line $1000 \mathrm{R}$ in a five column (A, B, C, D and E) by three row (1, 2 and 3) pattern as illustrated in Fig.1. The designation of the samples follows column number and row number, e.g. A1 refers to the sample made at the first column from the left (A) and first row from the back. During SLM, the laser moves among the three positions as indicated by red dots in Fig. 1 to cover the $400 \mathrm{~mm} \times 630 \mathrm{~mm}$ build area.

\section{Surface roughness}

Surface roughness of the SLM processed samples were conducted by a stylus contact method using a Mitutoyo Surftest SJ 410 surface profilometers. The stylus was set to traverse perpendicular to the layer boundaries, i.e., parallel to the build direction. The average deviation from mean-line of surface height Ra value is presented.

\section{Measurement of temperature during SLM using thermocouples}

Two oblique prism specimens with equal volume were designed for temperature measurement experiments. Both samples were built at a $45^{\circ}$ angle to the horizontal plane and has a cross-sectional dimension of $10 \mathrm{~mm} \times 15 \mathrm{~mm}$. The specimens were built to a certain height ( $15 \mathrm{~mm}$ from the base plate) and interrupted, then the powder particles were cleaned out (Fig.2a). The thermocouples were inserted and fixed to touch the inclined surfaces as shown in Fig.2b. Then the build chamber was filled with powder, while the thermocouples and the specimens were all buried in the powder bed. The process chamber is closed and refilled with Argon gas and the build plate was heated up to $100{ }^{\circ} \mathrm{C}$. Fig. $2 \mathrm{c}$ shows the finished build with powder cleaned away. The K-type thermocouples used for this experiment are $0.5 \mathrm{~mm}$ in diameter and possess a response time $\mathrm{t} 90 \%$ (time to reach $90 \%$ real temperature) of $0.06 \mathrm{~s}$. The data was recorded by a data-logger at a frequency of 1 reading per second. During the measurement phase, the laser was set to scan in unidirectional pattern along Y axis. The data channels are named after their surface orientations (upskin or downskin) and scan vector length $(10 \mathrm{~mm}$ or $50 \mathrm{~mm}$ ), and are pointed out by arrows in Fig.2c. The experiment was conducted on an EOS M280 machine.

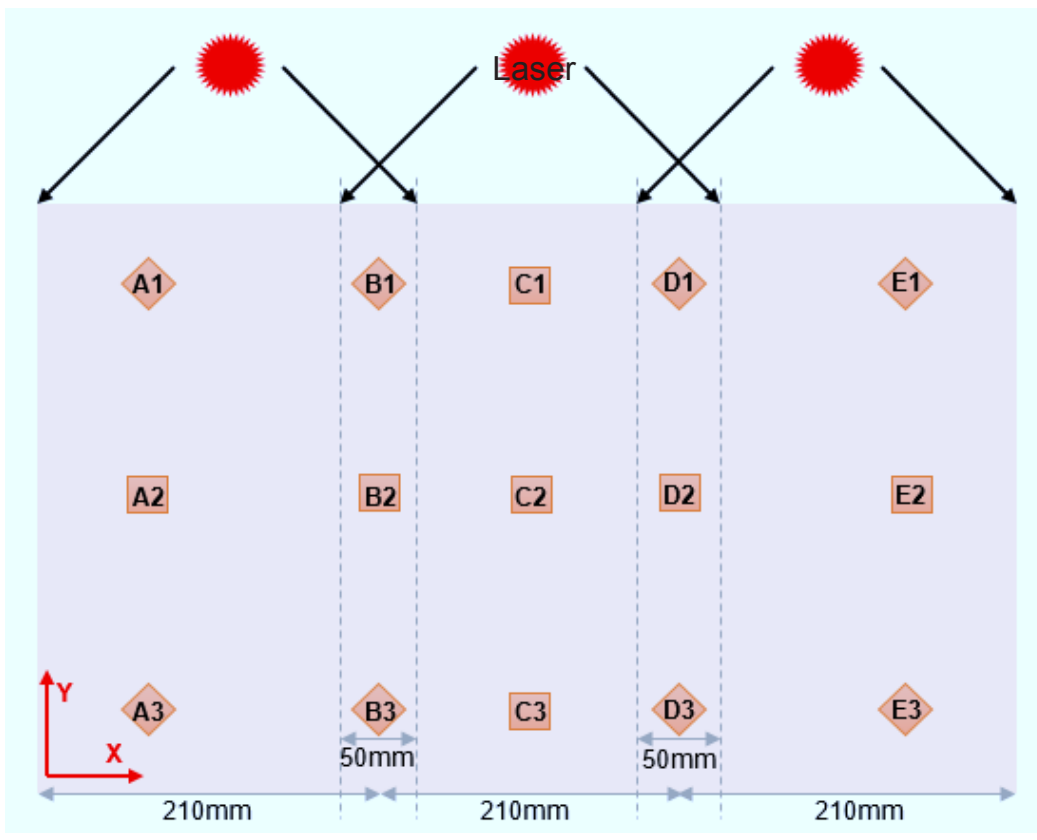

Fig.1 Sample arrangement on machine. the build platform of a Concept Laser X-line 1000R 

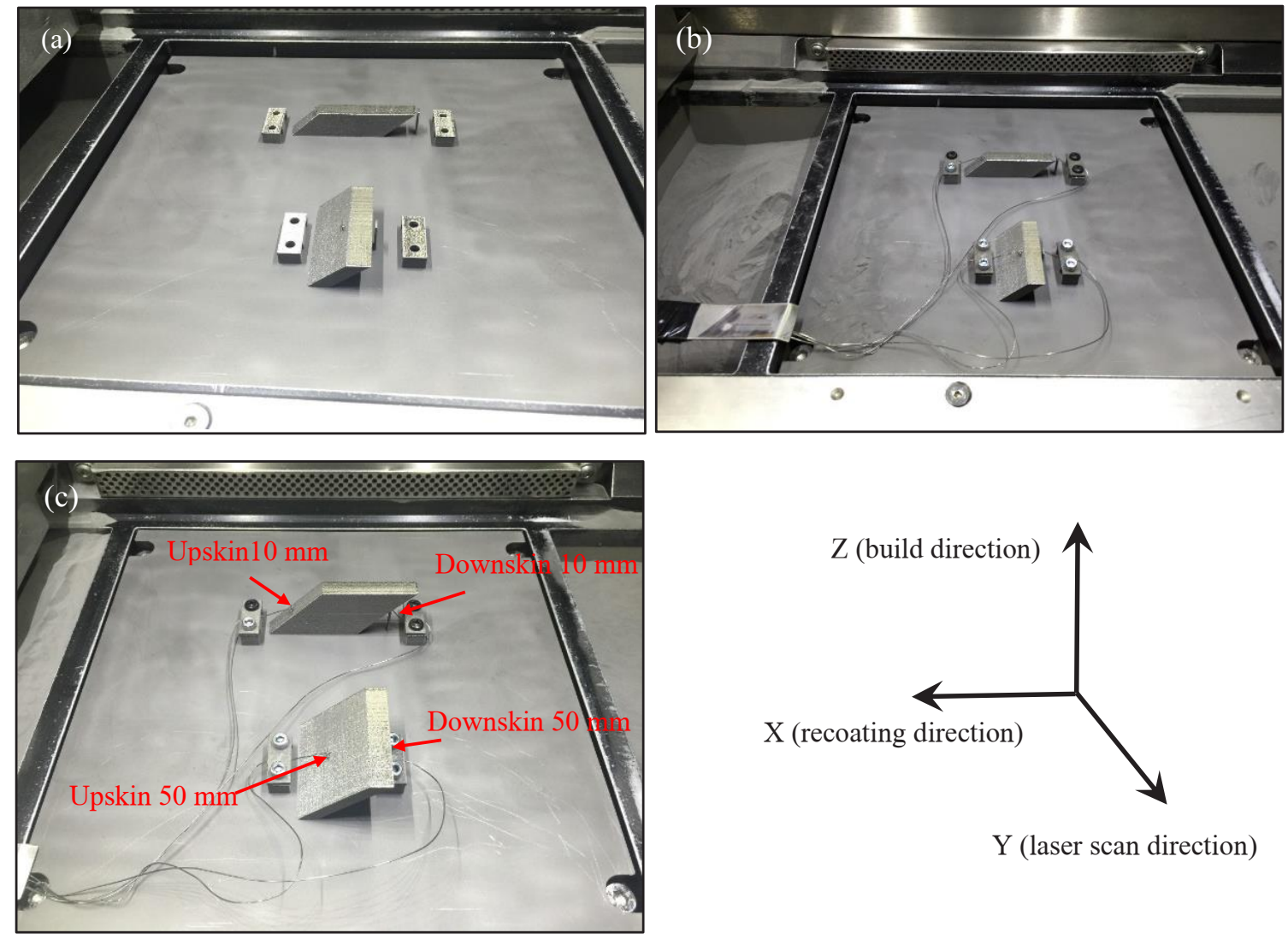

Fig.2 Experimental set-up for the measurement of temperature at part surface during the SLM process. 


\section{Results}

Surface roughness variation across the build

As shown in Fig.3a, the roughness values show strong effects of sample location. Samples in column B and D show highest means and standard deviations in Ra values while samples in column $\mathrm{C}$ show the lowest. Although sample locations in column A and column E are symmetrical about the centreline of the build plate, means and standard deviations of Ra values in column A are significantly higher than those in column E. There are three main sources of these variations in surface quality: (a) laser incidence angle, (b) overlap effect and (c) gas recirculation effect.

The farther away the samples are from the laser origin, the more the laser beam incidence angle at powder bed surface deviates from vertical incidence. As reported by Kleszczynski et al [12], laser beam incidence angle can result in excessive heat and melt extension into powder bed, thus creating rougher surface at particular sample surface orientation relative to the laser beam. It is evident in Fig.3a that samples made farther away from the laser origin shows higher roughness. For instance, as we compare the samples belonging to the same column, sample A1 and A3 are rougher than sample A2 that is slightly closer to the laser origin. Therefore we can infer from the results that smaller the incidence angle gets, the rougher the surface become as for the case of vertical surfaces studied.

In Concept Laser X-line 1000R machine, the laser is directed from three distinct locations above the build area depending where the part is located during the laser exposure. Hence the entire build area can be divided into three regions from left to right. Samples in column B and C reside in the narrow region where the three regions overlap with one another. For the exposure of the overlap regions, the laser beam deflection position alternates between the two nearest left and right positions. In other words, the samples in the overlap regions experience more variation in processing conditions in terms of laser incidence angle, which in turn increases surface roughness.

Fig.3c schematically shows the gas flow pattern, the inert gas is directed to make a turn on the top of the very left side of the build chamber. During the SLM process, the laser-powder interaction generates various kinds of by-products such as smoke that attenuates the laser beam and large spatter particles that are not easy to penetrate during the melting process. The effect of by-products is regulated by the local effectiveness of gas flow in removing the by-products, the higher roughness in column might be a result of local turbulence of inert gas flow.

(a)

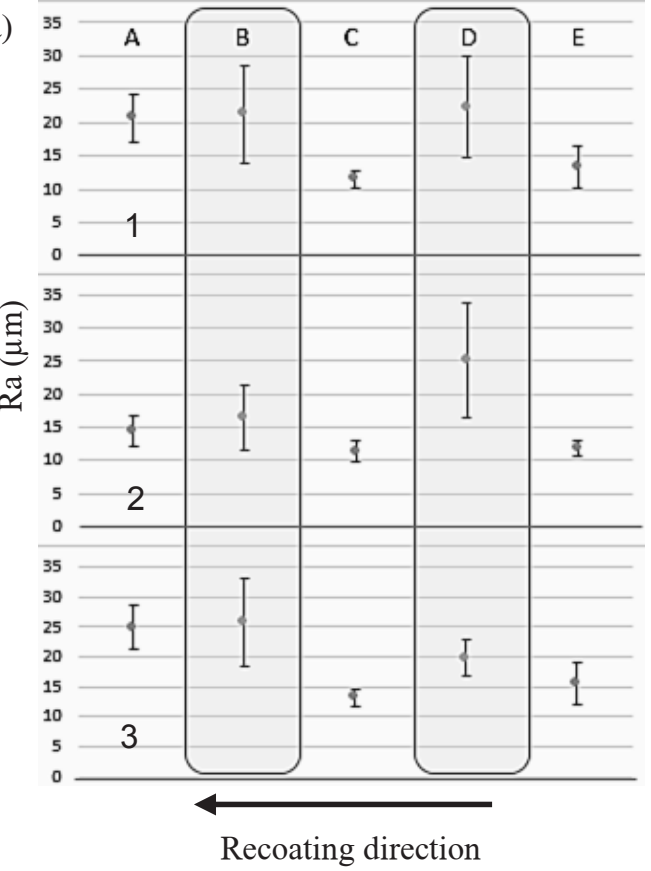

(b)

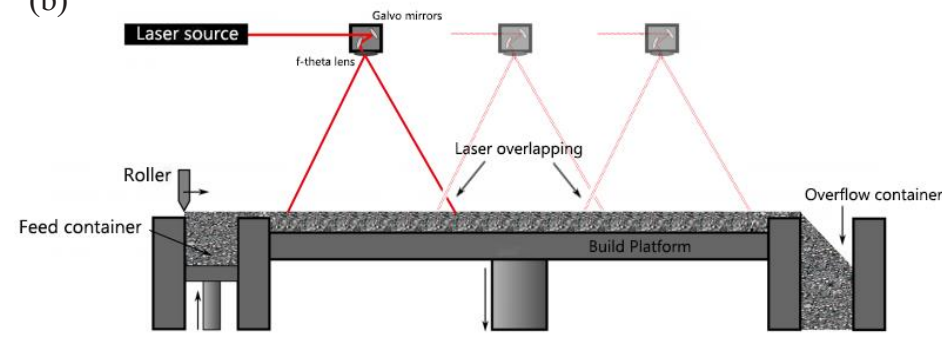

(c)

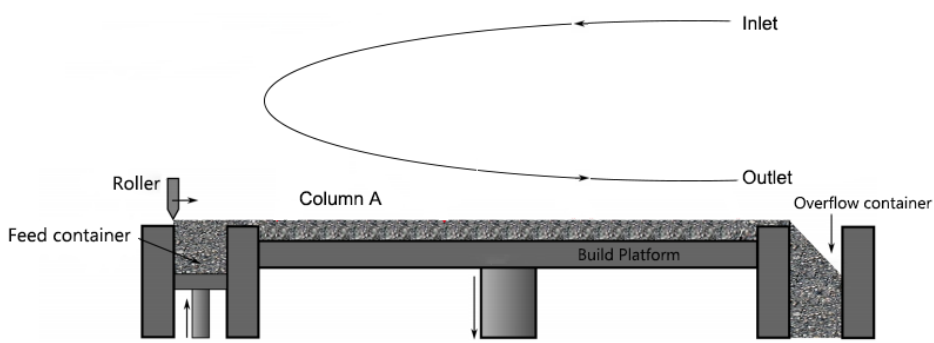

Fig.3 (a) Ra values of samples manufactured at various locations on the build plate; illustrative drawings of (b) three locations for laser beam deflection and (c) gas flow pattern in Concept Laser X-line 1000R.

Temperature evolution in oblique prism specimens

The thermocouples attached to the upskin and downskin surfaces of oblique prism experienced distinctively different heat transfer condition in terms of the proportion of solid metal in contact relative to less the thermally conductive powder. During the phase of platform re-heating to $100{ }^{\circ} \mathrm{C}$, none of the four channels reached $100{ }^{\circ} \mathrm{C}$ in two hours' time, while upskin surfaces 
reached slightly higher equilibrium temperature (Fig.4a). Since the thermocouples locate at a height below the currently exposed layer, the highest temperature recorded was far below the melting temperature of Ti-6Al-4V alloy. Channels at the down-skin surfaces showed much sharper peaks compared to those at the upskin surfaces. The temperature exhibited vibrant fluctuation as the build re-continued. The local fluctuations in temperature vanished with time and the temperature at the four channels exhibited a steady decline afterwards. The stabilised temperature range is determined by the heat transfer condition experienced by the respective channel, e.g. channel 'upskin $10 \mathrm{~mm}$ ' rose to the highest temperature during substrate heating and lost heat most quickly during the building process. On the other hand, overhangs (downskin channels) are prone to the issue of over-heating as heat are easily captured and accumulated due to the thermo-insulating effect of powder and lack of heat conduction pathways.

(a)

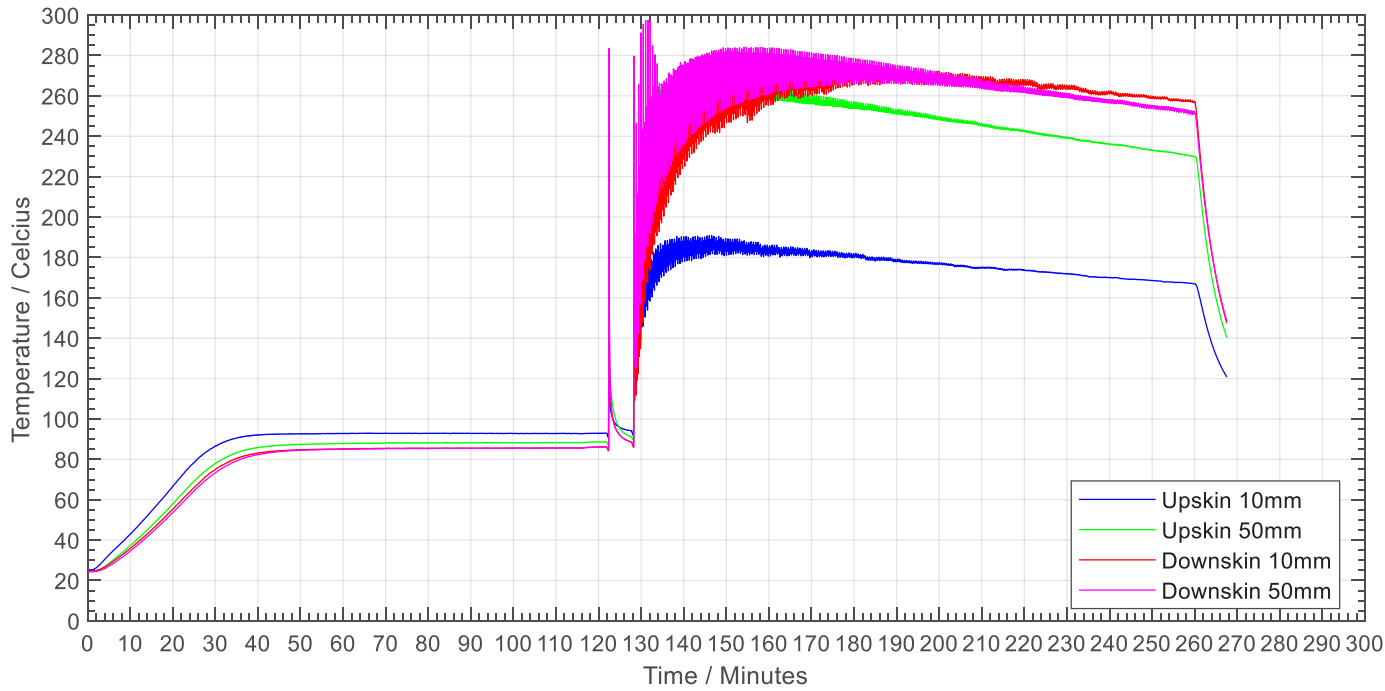

(b)

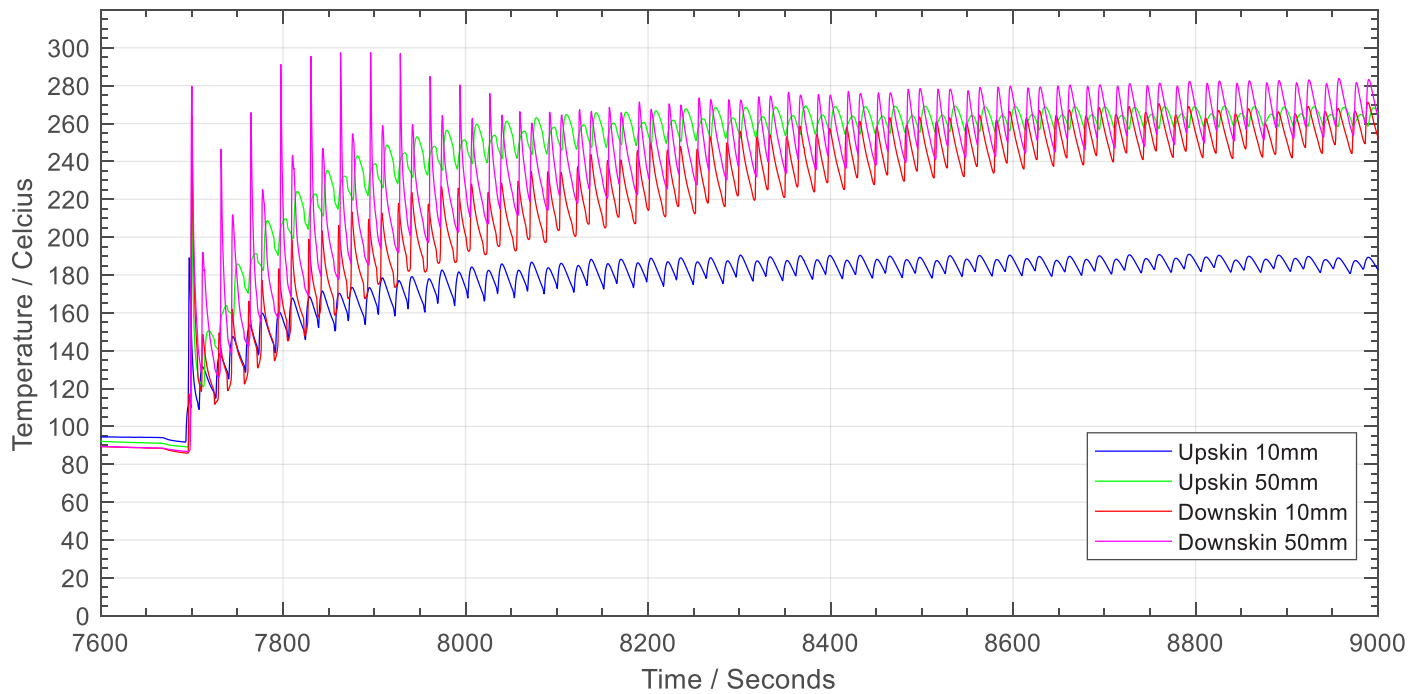

Fig.4 Temperature profile recorded by thermocouples fixed on upskin and downskin surfaces of oblique prism specimens. (a) Global temperature change including pre-heating, pre-exposure, and actual building process; (b) Zoomed in from (a) at the very start of the building process.

\section{Discussion}

The study in surface roughness is not only interesting for the reason that the roughness value itself is technically important but also for its implication for the variable processing conditions across the build envelop. Surface roughness of the SLM processed components is sensitive to many process-related phenomenon such as spatter generation, variation in powder size distribution, gas flow speed and direction, laser incidence angle, etc. [13]. In the present paper, the overlap issue as a hardware problem 
specific to Concept Laser X-line 1000R is analysed, it seems inevitable that one might encounter similar problems when multiple lasers or multiple gas inlet are installed for SLM machines with larger build envelop.

The experimental procedures of measuring the part temperature profile using thermocouple produces useful data for validation of part-level simulation of the temperature distribution, which has been published elsewhere [14]. It is also a complimentary approach to the developing online monitoring technologies which mainly focus on the temperature distribution of the very top layer exposed by the laser. The results suggest a strong dependency of local thermal history on the geometry of the part, the vicinity of the up-skin surfaces experiences faster heat transfer compared to the down-skin areas. As the SLM process progresses, the temperature stabilises to a range that is geometry-dependent. The layers on top of overhang areas are essentially building on a substrate of significantly higher temperature $\left(\sim 250{ }^{\circ} \mathrm{C}\right.$ as for the case of down-skin surfaces in Fig.4a). The differences in cooling rate and temperature of underlying layers (substrate) would both contribute to differences in solidification texture [15][16], grain size and morphology and mechanical properties.

\section{Conclusion}

The various sources of variations in part qualities during the SLM process was studied experimentally. Examination of surface roughness across the build reveals the specific regions of the build platform where quality issues are more likely to occur. Further works is needed to correlate specific surface features to the process errors. The in-situ temperature measurements using thermocouples fixated at surfaces of parts with different geometries imply the influence of powder as a thermal insulator and the effect of part geometry on heat loss. The local variations in thermal history has implications for part qualification where microstructural uniformity is of critical significance.

\section{Acknowledgement}

This research is funded by the Science \& Industry Endowment Fund under the program RP04-153 "Manufacturing a small demonstrator aero-engine entirely through additive manufacturing" and the Australia Research Council IH130100008 "Industrial Transformation Research Hub for Transforming Australia's Manufacturing Industry through High Value Additive Manufacturing”, including financial support from Safran Power Units and Amaero Engineering. The support and assistance from Monash Centre for Additive Manufacturing (MCAM) and Monash Centre for Electron Microscopy (MCEM) are gratefully acknowledged. 


\section{References}

[1] M. Mani, B.M. Lane, M.A. Donmez, S.C. Feng, P. Shawn, Int. J. Prod. Res. 7543 (2017) 1-19.

[2] C. Körner, E. Attar, P. Heinl, J. Mater. Process. Technol. 211 (2011) 978-987.

[3] Y.S. Lee, W. Zhang, in: Proc. Solid Free. Fabr. Symp., 2015, pp. 1154-1165.

[4] F.-J. Gürtler, M. Karg, K.-H. Leitz, M. Schmidt, Phys. Procedia 41 (2013) 881-886.

[5] C. Qiu, C. Panwisawas, M. Ward, H.C. Basoalto, J.W. Brooks, M.M. Attallah, Acta Mater. 96 (2015) $72-79$.

[6] S.A. Khairallah, A.T. Anderson, A.M. Rubenchik, W.E. King, Acta Mater. 108 (2016) 36-45.

[7] S. Berumen, F. Bechmann, S. Lindner, J.P. Kruth, T. Craeghs, Phys. Procedia 5 (2010) 617-622.

[8] T. Craeghs, S. Clijsters, E. Yasa, J. Kruth, Proc. SFF Symp. (2011) 212-26.

[9] M. Grasso, V. Laguzza, Q. Semeraro, B.M. Colosimo, J. Manuf. Sci. Eng. 139 (2016) 051001.

[10] C. Zhao, K. Fezzaa, R.W. Cunningham, H. Wen, F. De Carlo, L. Chen, A.D. Rollett, T. Sun, Sci. Rep. 7 (2017) 111.

[11] J.C. Fox, B.M. Lane, H. Yeung, Thermosense Therm. Infrared Appl. XXXIX 10214 (2017) 1021407.

[12] S. Kleszczynskia, A. Ladewigb, K. Friedbergerb, J. zur Jacobsmühlenc, D. Merhofc, G. Witta, Proc. 26th Int. Solid Free Form Fabr. Symp. (2015) 360-370.

[13] Z. Chen, X. Wu, D. Tomus, C.H.J. Davies, Addit. Manuf. 21 (2018) 91-103.

[14] M. Chiumenti, E. Neiva, E. Salsi, M. Cervera, S. Badia, J. Moya, Z. Chen, C. Lee, C. Davies, Addit. Manuf. 18 (2017) 171-185.

[15] M. Simonelli, Y.Y. Tse, C. Tuck, (2012) 480-491.

[16] A.A. Antonysamy, J. Meyer, P.B. Prangnell, Mater. Charact. 84 (2013) 153-168. 